\title{
Large Enchondroma of Metacarpal Bone with Pathological Fracture Treated with Marginal Resection and Reconstruction with Iliac Crest Graft: A Rare Case Report
}

\author{
Suresh Pandey ${ }^{1}$ \\ ${ }^{1}$ Chitwan hospital, Bharatpur, Chitwan, Nepal.
}

\begin{abstract}
Enchondroma is the benign bone tumor commonly found in phalanx and metacarpal shaft. The most common method of treatment is curettage with or without filling cavity with bone graft with recurrence rate up to $13 \%$. We present a large enchondroma of fourth metacarpal right hand with cortical breach treated with marginal excision and reconstruction of segmental defect with tricortical iliac crest bone graft with excellent functional and cosmetic result. We opted to report this case because of the large size of the tumor and successful reconstruction of metacarpal with simple technique of autograft.
\end{abstract}

Keywords: enchondroma; excision; metacarpal; reconstruction.

\section{INTRODUCTION}

Enchondroma is common benign bone tumor constituting about $3 \%$ of all bone tumors and about $12 \%-24 \%$ of all benign bone tumors. It is cartilaginous tumor arising from metaphyseodiaphyseal junction commonly phalanx and metacarpal bones due to proliferation of remaining hyaline cartilage from the medullary cavity. The common age group is second to fourth decade of life. Many patients are asymptomatic while some presents with pain, swelling, cosmetics or pathological fracture. The most common sites are proximal phalanx, middle phalanx, metacarpal and distal phalanx in descending order of its frequency. Small and asymptomatic lesion can be managed with wait and watch policy while larger painful lesion or with pathological fracture or cosmetic deformity needs treatment with curettage and filling the defect with bone graft. Recurrence rate after curettage and bone graft has been reported up to $13 \%{ }^{1}$

Very large lesion with pathological fracture or recurrent lesions are better treated with excision and reconstruction with autograft. We have reported this case of very large enchondroma with pathological fracture with extension to subchondral region of right $4^{\text {th }}$ metacarpal shaft treated with excision of the segment and successful reconstruction of metacarpal with simple iliac crest bone graft with excellent result. Informed consent was taken from the patient regarding use of data concerned for publication.

\section{CASE REPORT}

A 22 year lady, home maker by occupation, presented with progressive diffuse swelling over dorsum of right hand over $4^{\text {th }}$ metacarpal region for 6 month. It was painless to start with but she experienced mild pain on activity of the hand for 1 month. There was history of stiffness of the finger. There was no history of trauma or any other constitutional symptoms. On examination, there was ill-defined bony swelling arising from right $4^{\text {th }}$ metacarpal shaft with mild tenderness. There was loss of grip due to lack of complete flexion at $4^{\text {th }}$ MCP joint. There was no warmth over the swelling. Sensory and motor function was intact. Plain X ray revealed well defined bony swelling arising from $4^{\text {th }}$ metacarpal shaft extending to subchondral region of MCP joint with evidence of cortical breach. There was punctate calcification all around the tumor mass. There was no evidence of periosteal reaction. CT scan and MRI showed similar features without any soft tissue extension. Clinicoradiological diagnosis of large enchondroma with breach in the cortex with extension to subchondral region of the metacarpal head was made. Patient was explained about the disease nature, treatment plan and prognosis. Since tumor was very large with cortical breach with only wafer of cartilage left in the metacarpal head, excision of the segment along with lesion with reconstruction of the metacarpal with tricortical iliac crest graft was planned instead of just curettage and bone graft. She was operated under

Correspondence: Dr. Suresh Pandey, Chitwan hospital, Bharatpur-10, Chitwan, Nepal. Email: drsuresh.orthonepal@gmail.com. Phone: +977-9845047228. DOI:10.3126/jcmsn.v15i2.23275. Article received: 2019 -02-24. Article accepted:2019-05-30. 
Pandey. Large Enchondroma of Metacarpal bone with pathological fracture treated with..

general anaesthesia in supine position with dorsal incision with marginal excision of the tumor isolating and protecting extensor tendons and digital vessels and nerves (Figure 1).

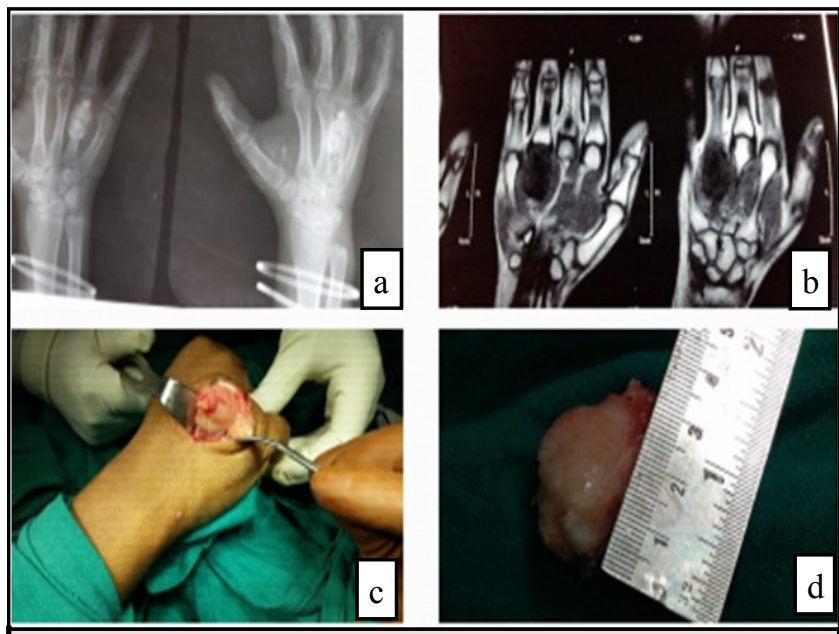

Figure 1. a) showing plain $x$ ray bony lesion from $4^{\text {th }}$ metacarpal extension to subchondral region of metacarpal head with multiple punctate calcification; b) MRI of the same hand showing expansile lesion arising from metacarpal shaft with extension to subchondral region proximally; c) Intraoperative photograph of the marginal excision of the tumor leaving proximal and distal end of metacarpal intact for reconstruction; d) the $3.5 \mathrm{~cm}$ excised tumor mass.

ipsilateral iliac crest. It was used to reconstruct the segmental defect of metacarpal and fixed with $\mathrm{K}$ wires. Haemostasis was secured and wound was closed with ulnar gutter slab application. Tissue was sent for histopathological examination and found to be enchondroma. There was no evidence of malignancy such as chondrosarcoma. Slab was removed in 2 weeks and ROM exercise was started.

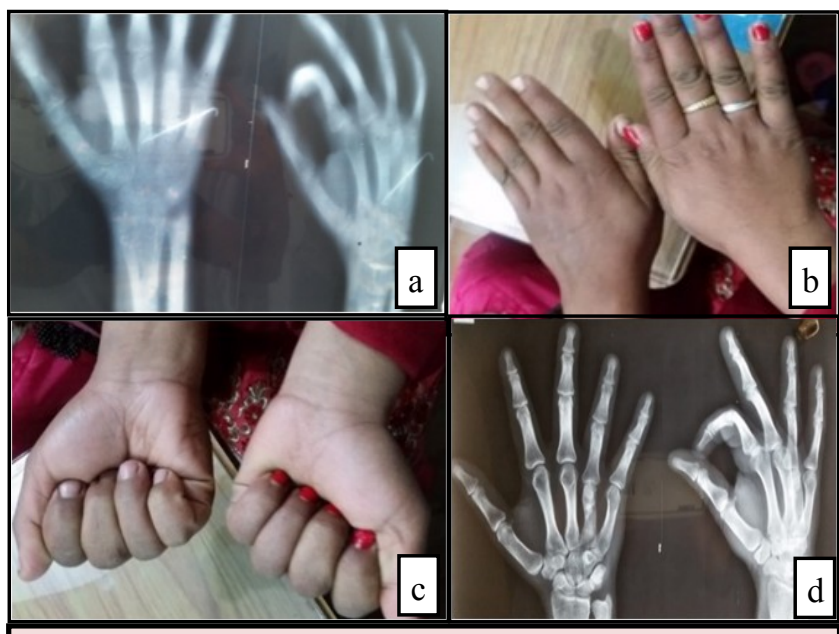

Figure 2. a) Postoperative segmental defect $4^{\text {th }}$ metacarpal right reconstructed with tricortical iliac crest graft and fixation with $\mathrm{K}$ wire ; b) and c) Range of motion at 3 month; d) graft union $X$ ray at 24 month D.
Pin was removed at 4 weeks. There was complete union of the graft at 6 weeks with full ROM and normal grip at 12 weeks (Figure 2).

There was no wound complication, pain or evidence of local recurrence till 24 months of follow up. Patient is satisfied with the result and there was no cosmetic problem. Written consent was taken from patient regarding use of these materials for academic publication keeping her identity undisclosed.

\section{DISCUSSION}

Enchondromas are the second commonest benign bone tumor next to osteochondroma and the most common benign bone tumors in the hand. ${ }^{2}$ It is cartilaginous in origin arising from mature hyaline cartilage. It can occur at centre of medullary cavity where it is called enchondroma or can be located eccentrically and is known as periosteal chondroma. The age group varies widely. ${ }^{3}$ Hand is the common site for this tumor with prevalence in proximal phalanx, middle phalanx, metacarpal, and distal phalanx in descending order of frequency. ${ }^{4}$ Treatment in symptomatic, large tumor or impending pathological fracture is curettage with or without filling the cavity. Small cavity with structurally insignificant can be left unfilled with hope to heal itself. But larger cavity needs filling with bone graft while large segmental defect or recurrent lesion needs reconstruction with graft to preserve the function and motion of fingers and hand. ${ }^{5-8}$

We treated this case with marginal excision instead of curettage as the size was large and is associated with cortical breach and extension to subchondral bone leaving only wafer of cartilage at metacarpal head to avoid chance of recurrence and preserve the function at the same time. There are reports of metacarpal and phalanx reconstruction with cement and hardware after segmental resection but we chose to reconstruct with iliac crest bone graft as it is more natural reconstruction. Reconstruction with bone cement and hardware has drawback of some loss of function of hand with stiffness in many cases. ${ }^{9}$ Yalcinka et al has reported successful reconstruction of $5^{\text {th }}$ metacarpal with pathological fracture with structural allograft in recurrence enchondroma treated initially with curettage and bone graft. ${ }^{1}$ Sassu et al has reported a large enchondroma of $3^{\text {rd }}$ metacarpal treated successfully with excision and reconstruction with vascularized fibular graft. ${ }^{10}$ Our case has been managed well with simple surgical 
Pandey. Large Enchondroma of Metacarpal bone with pathological fracture treated with..

technique of segmental resection of tumor and reconstruction of the defect with tricortical iliac crest graft resulting in full range of motion with normal grip without any cosmetic issue. The reconstructed graft united and remodeled in such a good manner that it resembled normal metacarpal

Conflict of interest: None.

Funding source: None.

\section{REFERENCES}

1. Yalcinkaya, M., Akman, Y.E. Bagatur, A.E. Recurrent metacarpal enchondroma treated with strut allograft: 14-year follow-up. Orthopedics.Volume 38, Issue 7, 1 July 2015, Pages e647-e650.

2. Sassoon, A.A., Fitz-Gibbon, P.D., Harmsen, W.S., Moran, S.L. Enchondromas of the hand: Factors affecting recurrence, healing, motion, and malignant transformation. Journal of Hand Surgery.Volume 37, Issue 6, June 2012, Pages 1229-1234.

3. Dorfman HD, Czerniak B. Benign cartilage lesions. In:Dorfman HD, Czerniak B, . Bone Tumors. St Louis:Mosby, 1998:253-276.

4. Kuur E, Hansen SL, Lindequist S. Treatment of solitary enchondromas of the hand. J Hand Surg 1989;14B:109-112.

5. Sekiya I, Matusi N, Otsuka T, Kobayashi M, Tsuchiya D. The treatment of enchondromas in the hand by endoscopic curettage without bone grafting. J Hand Surg 1997;22B:230-234.

6. Shimizu K, Kotoura Y, Nishijima N, Nakamura T. Enchondroma of the distal phalanx of the hand. J Bone Joint Surg 1997;79A:898-900.

7. Wulle C. The treatment of enchondroma. J Hand Surg 1990;15B:320 -330.

8. Bauer RD, Lewis MM, Posner MA. Treatment of enchondroma of the hand with allograft bone. J Hand Surg 1988;13A:908-916.

9. Jacob Bickels, James C. Wittig,Yehuda Kollender, Kristen Kellar-Graney,Kari L. Mansour. Enchondromas of the Hand:Treatment With Curettage and Cemented Internal Fixation. J Hand Surg 2002;27A:870-875.

10.Sassu, P., Burrai, S., Cara, L. A case of vascularized fibula bone graft in the treatment of a wide metacarpal enchondroma. Chirurgia. Volume 26, Issue 2, April 2013, Pages 125-128.

Citation: Pandey S.Large Enchondroma of Metacarpal Bone with Pathological Fracture Treated with Marginal Resection and Reconstruction With Iliac Crest graft: A Rare Case Report. JCMS Nepal. 2018; 15 (2):150-2. 